\title{
Biocompatibility and Toxicity of Magnetic Nanoparticles in Regenerative Medicine
}

\author{
H. Markides, M. Rotherham, and A. J. El Haj \\ Institute of Science and Technology in Medicine, Keele University Medical School, Thornburrow Drive, Hartshill, Stoke-on-Trent, \\ Staffordshire ST4 7QB, UK \\ Correspondence should be addressed to H. Markides, h.markides@istm.keele.ac.uk
}

Received 4 May 2012; Revised 28 June 2012; Accepted 16 July 2012

Academic Editor: Xiaoming Li

Copyright ( $) 2012$ H. Markides et al. This is an open access article distributed under the Creative Commons Attribution License, which permits unrestricted use, distribution, and reproduction in any medium, provided the original work is properly cited.

Regenerative medicine is a pioneering field aimed at restoring and regenerating the function of damaged cells, organs and tissues in order to establish normal function. It demands the cross communication of disciplines to develop effective therapeutic stem cell based therapies. Nanotechnology has been instrumental in the development and translation of basic research to the clinically relevant therapies. In particular, magnetic nanoparticles (MNPs) have been applied to tag, track and activate stem cells offering an effective means of monitoring in vitro and in vivo behaviour. MNPs are comprised of an iron oxide core with a biocompatible biological polymer. Safety is an issue of constant concern and emphasises on the importance of investigating the issue of toxicity. Any indication of toxicity can ultimately limit the therapeutic efficiency of the therapy. Toxicity is highly dependent on the physical, chemical and structural properties of the MNP itself as well as dose and intended use. Few in vitro studies have reported adverse effects of MNP on cells at in vitro in therapeutic doses. However, long term in vivo studies have not been studied as extensively. This review aims to summarise current research in this topic highlighting commonly used toxicity assays to investigate this.

\section{Introduction}

Nanotechnology is an emerging field with growing interest for its numerous applications ranging from information technologies to medicinal applications [1]. Subsequent social and economic implications of this field have resulted in its increased popularity and demand with a competitive drive [2]. It is heavily dependent upon the cross-collaboration of various scientific disciplines to manipulate and alter the dimensions of materials at an atomic scale resulting in the formation of nanomaterials [1]. Nanomaterials are defined as materials with one, two, or three external dimensions ranging from 1 to $100 \mathrm{~nm}$ - the nanoscale $[2,3]$.

Recently, nanoscale materials have been the centre of research, particularly in the fields of regenerative medicine and tissue engineering. Examples include nanoparticles, nanofibers, and nanotubes, all of which can be specifically tailored to their role and function within tissue engineering [4]. Nanoscale materials in these forms have been used in conjunction with stem cells to produce stem cell-based therapies with emphasis on replacing and restoring the function of cells, tissues, or organs in order to establish normal function
[5]. Examples include the transplantation of mesenchymal stem cells for the treatment of Huntington's disease [6]. This paper will focus specifically on the implications of applying nanoparticles in regenerative medicine and tissue engineering.

A nanoparticle can be defined as a material with three external dimensions of equal nanoscale dimensions [2]. The major benefit of using nanoparticles is that, due to their size, they can be accurately manoeuvred and targeted to a specific biological entity or marker [7] and interact on a cellular $(10-100 \mathrm{~nm})$, subcellular $(20-250 \mathrm{~nm})$, protein (3$50 \mathrm{~nm})$, or genetic scale $(10-100 \mathrm{~nm})[8,9]$. Their unique, electronic, optical, and magnet properties coupled with their specific dimensions have furthered their attractiveness in this field [10-12]. Furthermore, nanoparticles can be customised for a specific biological purpose such as cell isolation, drug delivery, diagnostics (magnetic resonance imaging MRI), cellular imaging, and hyperthermia $[8,11$, 13-15]. Examples of nanoparticles include quantum dots and magnetic nanoparticles (MNPs) $[12,16]$. Magnetic nanoparticles will be the main focus of this paper. 
1.1. Magnetic Nanoparticles (MNPs). Magnetic nanoparticles have a particle size within the nanoscale with magnetic properties. Various metals can be used to convey the magnetic properties of MNPs; nickel, cobalt, and iron have demonstrated to be such examples. Organic and inorganic polymers including RGD peptides, fibronectin, and dextran can be used to coat the magnetic core resulting in improved biocompatibility by protecting biological entities from adverse toxic reactions $[14,17]$. The customisation of surface coatings to desired specifications can be achieved via surface charge alterations, protein-binding capacity, and surface topography [18]; this increases functionalization of the particles encouraging enhanced interaction with biological entities, with minimal toxic effects $[8,11]$. Additional factors that can further influence the behaviour of MNPs are the size [19] and magnetisation (paramagnetic, ferromagnetic, ferromagnetic, and superparamagnetic) of the particles themselves [20].

Before nanoparticles can be considered for the use in regenerative medicine, they must fulfil the following specifications.

\section{Specifications:}

(i) biocompatible that is nontoxic to the cells [21-23],

(ii) biodegradable $[22,24]$,

(iii) maintain physical properties after surface modification [11],

(iv) must not affect stem cell characteristics [21],

(v) effective at therapeutic doses [21,22],

(vi) ideally have regulatory approval [20],

(vii) minimal or no transfer of by-products to surrounding tissue/cells [22, 25],

(viii) chemically stable in physiological conditions [24].

MNPs for the use in biomedical applications are desired to exhibit superparamagnetic properties (SPIONs) [26]. SPIONs are typically small particles composed of either a magnetite $\left(\mathrm{Fe}_{3} \mathrm{O}_{4}\right)$ or maghemite $\left(\gamma-\mathrm{Fe}_{2} \mathrm{O}_{3}\right)$ core [20, 27] coated with a biocompatible organic/inorganic polymer $[17,28]$ or precipitated throughout a porous biocompatible polymer $[10,20]$. Both maghemite and magnetite are traditionally ferromagnetic in nature. However, as they decrease in size to $30 \mathrm{~nm}$ or smaller, they lose their permanent magnetism and become superparamagnetic [2]. Iron ( $\mathrm{Fe})$ oxide-based MNPs are suitable for biological application for the following reasons: the superparamagnetic nature implies that the particles will not be attracted to each other, and so the risk of agglomeration in a medical setting is minimised [23]. Fe is a naturally occurring metal in the human body (ferritin), and hence iron-containing nanoparticles are biocompatible as the body is adapted to metabolising the particles into its elements; these can be utilised by the body in subsequent metabolic processes [19, 29-32].

The precedent for using SPIONs in regenerative medicine comes from the application of SPION-based magnetic resonance imaging (MRI) contrast agents [33] which are accepted T2 weighted contrast agents [26]. MR imaging is a safe and effective noninvasive means of imaging anatomical tissue [34]. It is thought that these contrast agents can be used for a variety of purposes in the clinical translation of stem cell-based therapies for the tagging, tracking, and activation of stem cells and other cell types [26, 29, 35]. Feridex (USA), also referred to as Endorem (EU) is example of FDA-approved, iron-based MRI contrast agent which has recently been taken off the market $[20,36]$. These particles have an iron oxide core and are between 50 and $180 \mathrm{~nm}$ in diameter with an overall negative charge along with biodegradable and biocompatible properties [20,32]. The dextran coating prevents the coagulation of particles; however a limitation to using dextran is its inability to enable the efficient uptake of these particles by cells. In these cases, transfection agents may be required.

The high resolution and impressive tissue penetration depth attributed to SPION-based MRI contrast agents has not only driven diagnostics to higher levels but also encouraged the multidisciplinary applications of these agents in fields such as regenerative medicine. These agents are, however, limited by their poor sensitivity thus preventing microscopic examination of tissue over time which is a crucial requirement in the development of stem cell-based therapies [12]. Other imaging modalities such as positive emission topography (PET scan), X-ray, ultrasound, and computed tomography (CT) [34] (available in a clinical setting) offer greater sensitivity in some cases but are lacking in other aspects such as the use of radioisotopes which could result in detrimental side effects in the case of PET scans [34]. This has therefore encouraged the integration of imaging modalities $[12,34,37]$ and, as a consequence, the development of multifunctional nanoparticles to be used in conjunction with various imaging techniques. In the context of regenerative medicine, this could further our understanding of biological processes related to stem cellbased therapies $[12,26]$. Unlike individual nanoparticles, multifunctional nanoparticles exploit the benefits of the imaging modalities of choice while minimising the disadvantages. In many instances this is translated in enhanced cellular tracking with high special resolution and high anatomical contrast without the issues of ionizing radiation for extended periods of time [12, 26, 37].

1.2. Multifunctional Nanoparticles. The development of multifunctional nanoparticles requires the reengineering and modification of particle surfaces to meet a greater variety of applications. It is highly desirable to incorporate fluorescent properties, near infrared absorption, and photon scattering, all of which enable in vivo imaging to a greater extent. This however is no easy task. It is a highly challenging task to incorporate the properties that make a particle a good MRI contrast agent and those that allow good NIR response, for example [12]. It involves novel and intricate approaches to achieve all the desired characteristics. In a study by Jin et al., the surface of nanoparticles was coated with gold (a noble metal) [12] in an effort to expand on the multifunctional aspect of MNP and incorporate near infrared absorption and photon scattering properties. This group successfully created smooth particles with the core spatially separated from the 


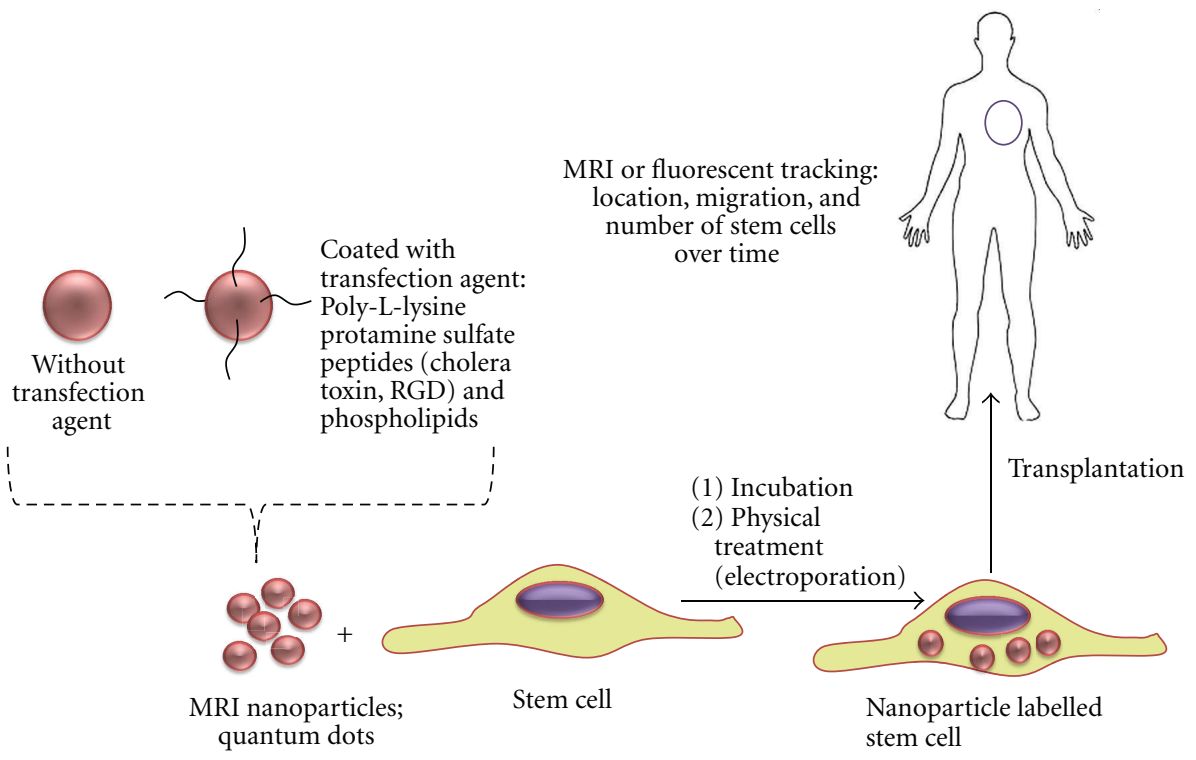

FIGURE 1: Schematic diagram highlighting the use of SPIONs in regenerative medicine. Cells requires the internalisation of SPIONS. Labelled cells are then implanted within the body and visualised by MRI [52].

shell thus resulting in uniform gold-coated MNP while still maintaining dimensions, electronic, magnetic, and thermal properties and still being responsive to NIR spectra [12]. Mahmoudi et al. took this one step further and created goldcoated magnetic nanoparticles with jagged edges to allow for ramen spectroscopy to be employed and in this way imaging at the molecular level [26]. In another study, Mahmoudi and Shokrgozar sought to add to the multifunctionality of these particles by adding and trapping a fluorescent polymeric dye between the core and the gold coating [37]. This simple addition adds an extra imaging modality for either in vivo or in vitro imaging.

1.3. Application of SPIONS in Regenerative Medicine. SPIONS are particularly useful and have a multifunctional aspect within regenerative medicine where their functions include tagging, tracking, and activation of stem cells both in vitro and in vivo;this multifunctionality makes them highly desirable tools in this field.

1.3.1. Tagging. Incorporating SPIONs into cells allows for the remote manipulation of cells using an external magnetic field gradient. This encourages the precise positioning or targeting of cells to desired sites for tissue regeneration or repair $[45,46]$, allowing them to function as a powerful, noninvasive tool in stem cell therapy $[47,48]$.

1.3.2. Tracking. A universal issue central to all cell-based therapies is the in vivo migration of stem cells within tissues and on substrates, which is often guided by the presence of chemical mediators and material topography [49]. The migration of these cells to and from the target location requires precise monitoring to determine the effectiveness of the therapy. The use of nanoparticles can provide answers to questions such as optimal delivery route; extent of engraftment; migratory patterns after transplantation; ideal dosage schemes. Having the answer to these questions will help in the optimisation of the overall therapy and thus increase its therapeutic potential [50]. It is thought that MNP can be employed in conjunction with the use of magnetic resonance imaging (MRI) to track implanted cells in vivo (Figure 1). In essence, stem cells are encouraged to internalise iron-based MNP; in this way magnetic properties are transferred to the cells. The intracellular iron essentially disturbs the local magnetic field thus allowing cells to be visualised as a lack of signal with MRI [51].

1.3.3. Activation. Mechanical stimulation can be used to facilitate cell proliferation, differentiation, and migration of stem cells [53]; physical forces include fluid flow, axial compression, tension, and magnetism [54]. Biological responses are achieved through the process of mechanotransduction, whereby cells convert physiological mechanical stimuli into biochemical signals to activate the biological response [55]. Using MNP functionalised with antibodies or peptides, it is possible to attach SPIONS to specific mechanosensitive cell surface receptors and ion channels. This has been shown to result in membrane polarisation, receptor activation, and subsequent downstream second messenger signals in hMSC [56]. Using this technology it has been shown that it is possible to promote an osteochondral phenotype of hMSC and human osteoblasts in response to magnetic activation using an external oscillating magnetic field $[57,58]$.

1.3.4. MNP-Mediated Transfection. The transfection of therapeutic genes could play a pivotal role in regenerative medicine. This technology allows the replacement of defective genes or addition of extra copies of therapeutic genes that are known to play a crucial part in the regeneration of tissue. One example is the treatment of ischemia by 
transfecting human umbilical vein endothelial cells with vascular endothelial growth factor to promote upregulation of survival factors and subsequent improved cell viability [59]. There are many mechanisms of inserting exogenous genetic material into cells. Transfection with viruses can be an effective technique but there are safety and efficacy concerns with this strategy. Nonviral transfection methods, for example, electroporation, transfection reagents, are considered safer. However these mechanisms often suffer from poor transfection efficiency or impact on cell viability to unacceptable levels depending on cell type. Therefore, a stable, high transfection system with minimised toxicity that can successfully deliver genes of interest without compromising gene function is required [60]. MNPs are currently being considered as alternate gene delivery vehicles; coated with nucleic acid MNPs have shown the capacity to further increase transfection efficiencies using magnetic fields to attract the MNP towards the cell membrane; the particles are then taken up by the cells by various uptake mechanisms such as endocytosis. Upon uptake the DNA dissociates from the nanoparticle and can then be expressed. One study has successfully transfected the MG63 cell line without any major effects on cell viability [61].

To summarise, all three applications require the labelling of stem cells with SPIONs. This can be achieved through either the internalisation of SPIONs or the binding of SPIONS to cell surface markers, for example, integrins, or to specific antibodies $[50,62,63]$.

\section{Safety and Biocompatibility of Magnetic Nanoparticles}

Toxicity issues are a major concern and are important factors in the context of regenerative medicine and tissue engineering. As mentioned previously, the use of MNPs in regenerative medicine requires the labelling of cells (the therapeutic agents) with MNPs which may then be implanted within the body. Employing particles which are toxic in nature over a long period of time can significantly diminish the therapeutic efficiency of the cell-based therapy [38]. It is valid at this point to state that toxicology is defined as the study of adverse effects of chemical, physical, and biological agents in people, animals, and the environment [64]. Toxic cellular effects are translated into impaired mitochondrial activity, membrane leakage, and morphological changes. This can have adverse effects on cell viability, proliferation, and metabolic activity and impair the therapeutic efficiency of the therapy [65]. In cases where the MNPs are incorporated into the therapy and transplanted within the body, the risk of MNPs migrating through the organism, entering, and accumulating within organs is a constant concern. This could trigger an immunological or an inflammatory response by the body $[2,17,64]$. These are all highly undesirable consequences. Labelling stem cells for this application therefore demands the preservation of physiological cellular properties and the retention of MNPs over prolonged periods [66]. This formulates the following question: would any of the properties related to MNPs indicated for regenerative medicinal use provoke an adverse effect on either the cells (in vitro applications) or the body (in vivo applications)?

The toxicity of MNPs on biological entities is highly dependent on a range and combination of factors related to the properties of the MNP itself; structural properties, dosage, and the intended use are among dominating factors $[38,64]$. The chemical composition of the particles themselves can be naturally toxic. Interestingly, some materials which are known to be inert in large quantities are in fact toxic at nanoscale, such as gold [64]. It has been found that certain metals such as cobalt, nickel, cadmium, zinc, and silver are toxic to biological entities and therefore not suitable for biomedical applications while others such as titanium and iron oxide-based particles are considered significantly less damaging to cells $[17,30,33]$. In the case of cadmium selenide (CdSe), cadmium ions demonstrated to cause cell death in primary hepatocytes [38]. Additionally the location of MNPs in relation to the cells is an extremely important consideration [38]. For instance, MNPs could invoke a cytotoxic response when internalised by potentially interfering with the biological function of the cells but not when attached to the cell membrane [11, 38]. In other cases however, SPIONs attached to the surface of cells may interfere with cell surface interaction [11]. Furthermore, the physical properties such as the particle size, shape, and surface coating can also evoke a toxic response by aggregating and coagulating according to size and shape [38, 64]. When addressing the possible in vivo application of MNP, it is equally important to consider the fate of the MNPs after they have been released by the cells. Further issues arise when considering the degradation of the MNPs and the outcome of accumulated MNPs or MNP by-products in various tissues and organs [2]. Degradation products are thought to possibly react with various components of the body or cells. Therefore, the effects of the breakdown products on the surrounding tissue should be fully investigated [2, 29]. Therefore assumptions on this matter cannot be made, and appropriate investigations should always be performed.

At the cellular level, oxidative stress is thought to be the main cause of toxicity by MNPs [40]. Oxidative stress arises when there is an imbalance between damaging oxidants also referred to as reactive oxygen species (ROS) such as hydrogen peroxide, hydroxyl radicals, and the protective antioxidants of which vitamin $\mathrm{C}$ and glutathione are examples. ROS are primarily formed by the incomplete reduction of oxygen [67] The accumulation of oxidants eventually leads to destruction of cellular proteins, enzymes, lipids, and nucleic acids, and as a consequence the normal cellular processes become impaired leading to the development of diseases and cell apoptosis and necrosis [17, 23, 40, 67]. ROS can be generated from the surface of MNP, the leaching of metal ions from the core, or release of oxidants by enzymatic degradation of the MNP [2]. ROS production can be measured using dichlorofluorescein diacetate fluorescent probe after SPION administration [65]. It has been reported that dissociated iron oxide MNP can promote the formation of ROS and hydroxyl radicals, and as a result may lead to cellular toxicity along with impaired cell metabolism and increases in 
apoptosis [11]. On the other hand, ROS can be used in cancer therapies to destroy damaging cancer, for example [67].

\section{Studies}

Years of research have demonstrated the ability of SPIONs to be taken up by a wide variety of cells by simple incubation. Cell types include fibroblasts, lung cells, liver cells, stem cells, kidney cells, macrophages, nerve cells, endothelial cells, and various cancer cells lines [2]. There is also an impressive portfolio of MNPs that have been used with a variety of cell types. The degree of toxicity has been known to vary with cell type, MNPs, and a combination of these two factors. It is therefore essential to carry out toxicity studies to evaluate the toxicity of specific MNP on the particular cell type of interest [29]. For instance, it has been reported that uncoated SPIONS caused significant cell death in dermal fibroblasts while lung cells appeared not to be affected [2]. This highlights the importance of cell type and MNP relationship. The general consensus is that labelling cells with SPIONs is safe validated by the viability, proliferation, and differentiation capacity of cells being unaffected thus justifying its use in regenerative medicine $[20,29]$. Other studies have shown that internalised silica-coated magnetic nanoparticles are biocompatible with stem cells [38]. Silica is a particular beneficial coating for nanoparticles since it can easily be functionalised, and it is resistant to degradation within a cellular environment whilst still being biocompatible $[38,40]$.

Toxicity is investigated through a series of in vitro and in vivo experiments, following the general schema below (Figure 2). In vitro toxicity tests offer a quick and simple means of gathering preliminary toxicity data which is also cost effective with minimal ethical issues [17]. When carrying out in vitro investigations, it is essential to identify and apply reproducible in vivo environments in terms of expanding conditions and sample preparation in vitro [2]. Data revealing marginal or no toxicity via in vitro tests can then be moved onto in vivo studies [68]. In these situations, small animal experiments are carried out and monitored over time to investigate the long-term effects of MNPs in a biological setting. Toxicity validation tests include histology on injection sites and major metabolic sites (liver, pancreas, kidney, brain) to look for signs of MNP spreading and accumulation. Sections are stained for iron by prussian blue stain and caspase 3 as an indicator for apoptosis within these areas [69]. It is not unlikely to have contradicting in vitro and in vivo results. This may be attributed to in vivo bodily functions/processes such as homeostasis, working to expel foreign reagents which are not present in vitro, for example, kidneys acting to filter blood [17]. Should in vivo studies reveal encouraging results, both the therapeutic efficiency and the safety of the MNP can be fully evaluated, and the MNP treatment will have to be approved for clinical use by regulatory bodies such as the Food and Drug Agency (FDA). This requires human clinical trials. An important question is whether the particles are regulated in combination with the therapy or independently. This question highlights the fact that the regulatory framework relating to these particles is limited, thus making it difficult to evaluate the safety of the

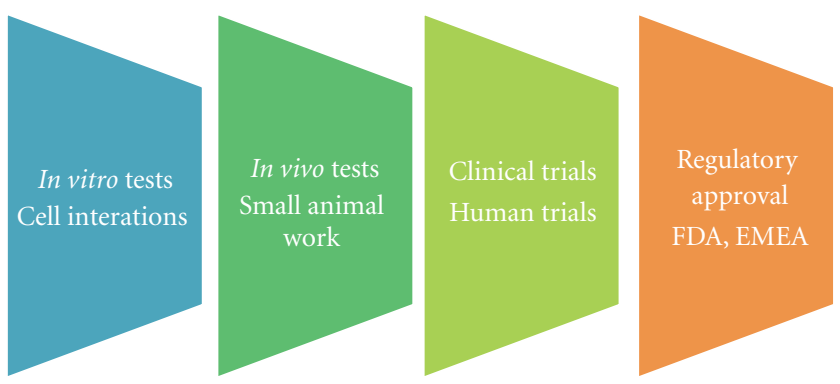

FIGURE 2: Schematic highlighting the route of translating stem cellbased therapies (incorporating the use of MNP) from bench to bed side.

nanomaterials in conjunction with regenerative medicine. For this reason, extensive safety assessment of these particles must be carried out to satisfy not only the regulators but also the patient. Even though SPION-based MRI contrast agents have been used and approved, the use of these for stem cell-based therapies still requires FDA approval as their intended use is different from their use as contrast agents [36]. An essential requirement of contrast agents is to be excreted relatively quickly and not remain in the body in the long term. However, for some applications, especially within the remit of regenerative medicine, long-term treatment of MNPs and presence in the body may be necessary. Using FDA-approved contrast agents will however make this task easier [66].

Table 1 highlights commonly used in vitro tests for MNPmediated toxicity and cell viability. It can be concluded that MTT (3-(4,5-Dimethylthiazol-2-yl)-2,5-diphenyltetrazolium bromide), PI (Propidium Iodide), for example, live/dead assay [70] and trypan blue [71] stains are most commonly used. Other popular tests not mentioned in this table include BrdU (5-bromo-2'-deoxyuridine) assay and the LDH (Lactate dehydrogenase) assay for metabolic activity [10]. Toxicity assays are aimed at investigating vital cellular activity such as cell death and cell viability. Cell death or apoptosis is most commonly assessed using the tetrazolium compound MTT [72, 73], LDH [74], and PI [75] assay while cell proliferation is assessd using BrdU [76] and MTT [73]. Figure 3 describes each test in greater detail. Other notable cytotoxicity assays include cell-life cycle assay, TUNEL assay (i.e., for apoptosis detection) [77], and various redox assays [78]. Immunochemistry can also be performed to look for markers of apoptosis or necrosis [79]. Furthermore, many toxic effects caused by MNP may stop mitochondrial activity which is measured by assays such as MTT. Added to this, there are inherent issues associated with MNP-cell interaction, dosage, and time course, all of which may impact on results [79]. It should be noted that in vitro tests that produce specific and quantitative toxicity read-outs are particularly convenient for the initial evaluation of toxicity and biocompatibility of new MNP [79]. An important consideration when interpreting the results of these types of in vitro assays is that they often provide little information on the mechanism of toxicity or the cause of cell death. In addition it can be noticed that SPIONs do not affect cell viability, proliferation, or differentiation capacity of stem 


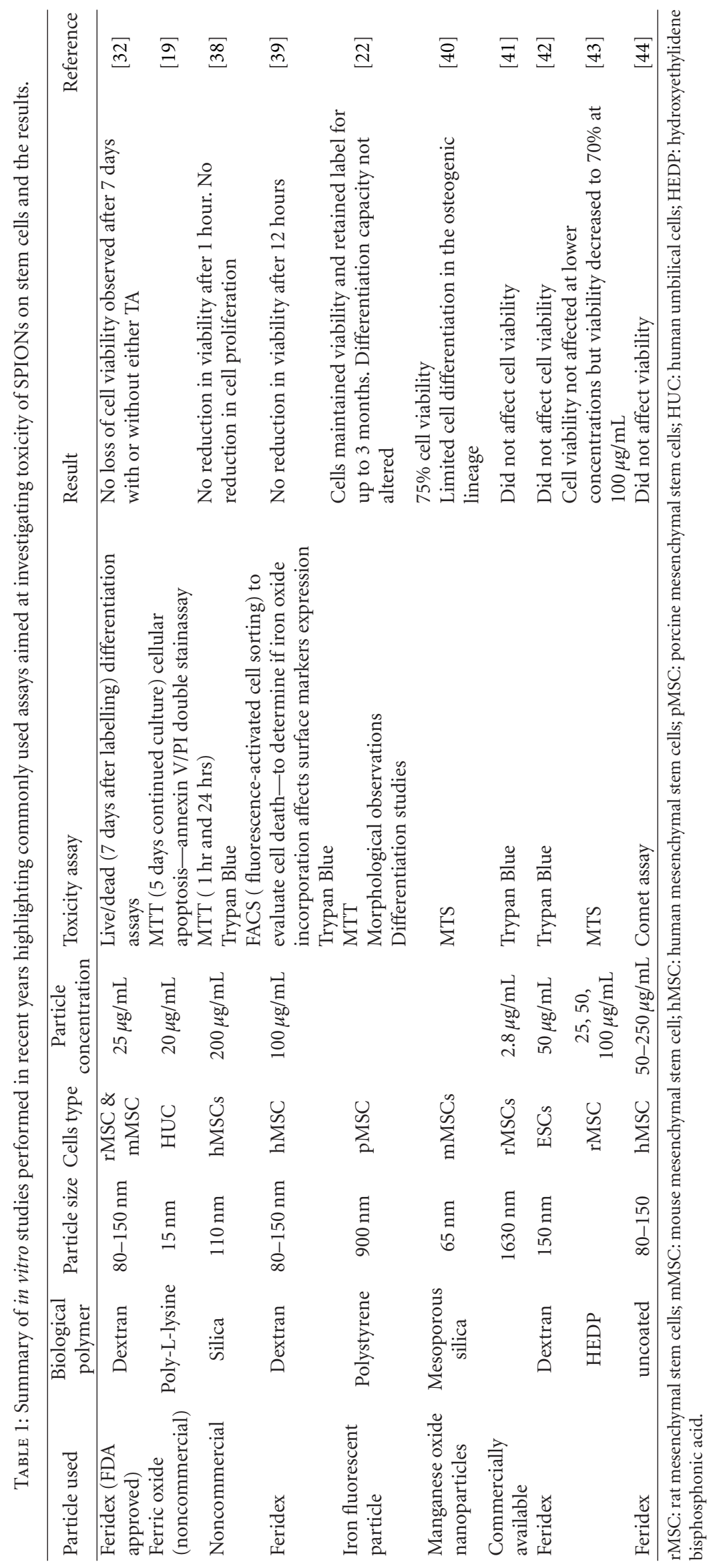




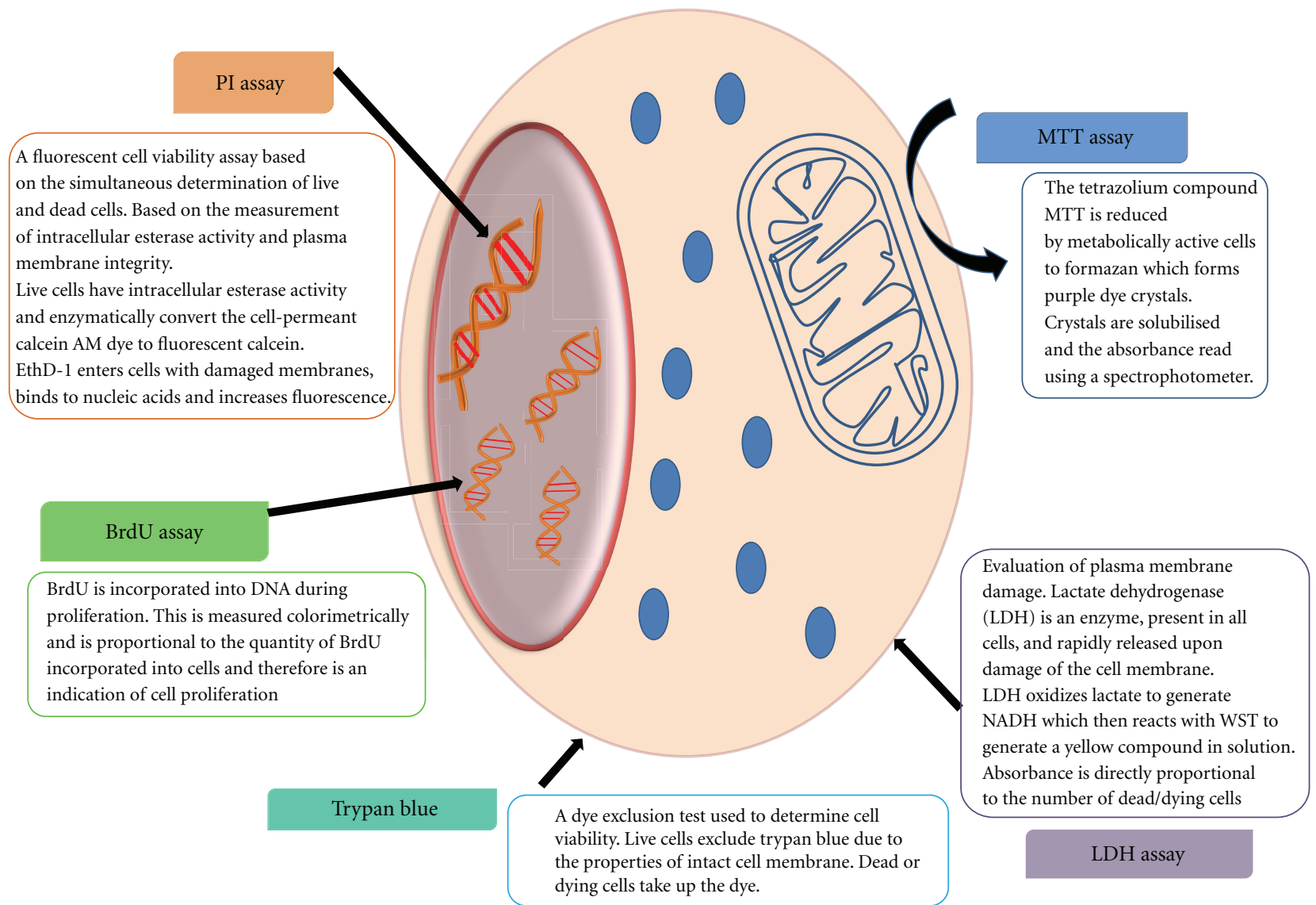

Figure 3: Overview of in vitro toxicity assays. Cell image adapted from [2]. PI [75], MTT [72, 73], LDH [59], Trypan Blue [71] and BrdU [76].

cells. In fact it can be said that number of studies reporting adverse or toxic effects of MNP are few [17].

Despite cost, time, and ethical considerations, in vivo tests in relevant animal models are crucial for the study of biological effects that cannot be modelled in vitro. This includes the pharmacokinetics of MNP in the body, that is, absorption, distribution, metabolism, and elimination. These studies allow information about the systemic toxicities to be elucidated including immunological, neurological, reproductive, cardiovascular, reproductive, and developmental as well as any carcinogenic effects [79].

As far as in vivo assays are concerned, one of the important functions to assess is the blood contact properties of the MNP, that is, blood compatibility. In terms of in vivo application of MNP, should the MNP be incompatible with bio fluids such as blood, this could lead to haemolysis, coagulation, and blood clots due to adsorption and/or activation of biomolecules, for example, plasma protein, complement factors, and so forth. As such it is critical to assess these properties before clinical use. One such test is the haemolysis assay which uses erythrocytes to assess the MNP toxicity. The coagulation tendencies can be assessed using common clinical assays including prothrombin time (PT), activated clotting time (ACT), and activated partial thromboplastin time (APTT) [79]. Another parameter to assess is the inflammatory response. This can be evaluated by looking at levels of chemokines and proinflammatory cytokines, for example, IL-8 in the blood as these can indicate potential oxidative stress toxic effects caused by MNP [34]. Gene expression analysis for transcription factors associated with oxidative stress can also be examined and may reveal useful information about the mechanism of toxicity. This raises the possibility of correlating MNP characteristics with toxic effects observed in certain cell lines and tissues [79].

Once the MNPs have entered body, MNPs come into direct contact with biological macromolecules such as proteins, lipids, and enzymes found within the biological fluid. These biomolecules interact with the surface of the MNP to form a complex layer of molecules over the MNP. This biological layer is termed "corona" $[80,81]$. The physical and chemical properties of MNP (size, surface coating, and functionalization) dictate the binding of molecules to the particles resulting in either a hard or weak corona which is significantly different to that of the surface of the MNP $[80,81]$. This affects the initial interaction of the cell with the MNP as the cell's first point of contact is with the corona and not the surface of the particle [80]. In turn, this dictates subsequent cellular and tissue responses [81]. This protein corona could be responsible for various toxic outcomes [80]. The formation of the corona is a dynamic process, and competitive process involving the attachment and detachment of various proteins as the particle moves from one biological 
environment to another [81] Movement of particles from one biological environment to another causes proteins from original fluid to be replaced by proteins from new fluidleaving evidence of the previously attached proteins [80, 81]. Approximately 10-50 proteins with the highest affinity for the surface are bound at one time [81]. The parameters affecting MNP-protein interaction include physiochemical properties (such as surface chemistries, particle size, shape, charge, surface area, surface defects, smoothness/ roughness, and functional groups) of MNP and the composition of biological fluid (proteins types and ratios) [81].

The interaction of proteins with the surface of MNPs of different types and coatings is also an important consideration when assessing in vitro toxicity. When MNPs are added to culture media, media proteins and other nutrients may adsorb onto MNPs and thus are unavailable for cellular activities. This naturally has implications on cell growth and viability. It is paramount therefore to test different culture media as MNP-protein interaction is dependent on the in vitro environment and MNP composition, so different media recipes could influence the outcome of cytotoxicity assays $[78,82]$.

A related concept is the effect of "cell vision" presented by Laurent et al. [82]. This refers to the contact point between MNP and the cell membrane, which is characterised by its surface molecules, that is, proteins, sugars, and phospholipid composition. The binding of MNP to these structures which may be different for different cell types defines how individual cell lines "see" MNPs. As a result the binding of exogenous objects like MNP to these structures may cause different responses and influence uptake and metabolism depending on the cell type. This concept is exemplified by Laurent et al. who showed that the same concentration of SPIONS can cause significant toxicity on neuronal and glial cells whilst displaying little toxicity on other cell types like heart and kidney cells $[82,83]$. The issues of cell vision and MNP-media interactions raise questions about the reliability of toxicity assays when studying the toxic effects of MNP. This issue is highlighted by the seemingly contradictory results of some MNP toxicity assays [34]. To address this issue Mahmoudi et al. have modified the MTT protocol by exposing SPIONS to culture media thereby allowing MNP to interact with culture media proteins before adding them to cell cultures. This led to improvements in the reliability of in vitro MNP toxicity results. Their results showed that uncoated and coated SPIONs were less toxic as previously thought and induced toxicity in various cell lines at greater doses than permitted for humans [78]. Furthermore another problem is posed by the sedimentation of MNPs, as this phenomenon effectively leads to an apparent increase in MNP concentration at the cell surface and so should be taken into account when performing toxicity assays [82].

\section{Conclusion and Future Perspectives}

It has been mentioned that in vitro and in vivo toxicity results often contradict each other. This can be attributed to the issue of protein adsorption onto the surface of MNP. It has also been suggested that various biomaterials intended for biological/tissue engineering purposes may benefit from exposure to biological fluids prior to implantation. This would allow for a layer of preadsorbed proteins to adhere on the surface of the material which may offer several advantages in terms of cellular mediated responses [84]. Following this theory, in vivo toxicity studies could be mimicked to a closer extent in vitro with the MNP in question when exposed to the desired biological fluid.

The multifunctional applications of MNP have been established. However utilizing micro- or nanoporous structures with nanometer superparamagnetic particles embedded within this structure could offer an alternative means of activating and inducing differentiation of stem cells for tissue engineering in vivo. Various biocompatible and bioactive materials have been used to fabricate these porous structures and have had impressive results in terms of cellular compatibility and differentiation in terms of osteogenesis and chondrogenesis $[84,85]$. Examples of such porous nanoparticles include nickel titanium nanoparticles coated with titanium oxide [85] and calcium phosphate ceramics [84]. Preexposing nanoparticles to solutions of specific proteins could also warrant increased cell attachment, proliferations, and the differentiation of stem cells down various lineages such as osteogenic or chondrogenic pathways [84, 85]. Thus combing the use of biocompatible materials known to promote or induce differentiation with magnetic properties could be of great benefit in tissue engineering and regenerative medicine.

This paper has addressed the relevance of MNP in regenerative medicine, highlighting their diverse and significant applications within this field. MNPs offer a noninvasive, practical means of monitoring, controlling, and targeting stem cells to optimise the therapy with clinical adoption in mind. However, safety concerns could ultimately prevent the adoption of MNP in regenerative medicine. This would demand the development of alternative ways of monitoring and controlling stem cells in vivo. It is essential to confirm the safety of the procedure prior to in vivo transplantation despite clinical efficacy of the technique. In reality, there is an enormous portfolio of particles available both commercially and in research. This makes it very difficult to give a definitive answer to the following question: are MNPs toxic? The toxic effects of MNPs should be evaluated for each specific purpose. A large number of in vitro toxicity investigations have shown no adverse side effect of labelling stem cells with SPIONs. However, long-term in vivo studies have not been studied as extensively and hence are an area of much needed research.

\section{Acknowledgments}

The authors would like to thank D. Kumar for his contributions to this work. Support was received from the EPSRC Doctoral Training Centre (EP/F/500491/1) and LOLA BB/G /010579/1.

\section{References}

[1] M. Chakraborty, S. Jain, and V. Rani, "Nanotechnology: emerging tool for diagnostics and therapeutics," Applied 
Biochemistry and Biotechnology, vol. 165, no. 5-6, pp. 51178 51687, 2011.

[2] H. H. Morteza Mahmoudi, R.-R Barbara, and P.-F Alke, Assessing the In Vitro and In Vivo Toxicity of Superparamagnetic Iron Oxide Nanoparticles, American Chemical Society, 2011.

[3] J. Wang, Y. Gao, and Y. Hou, "Evaluation on cartilage morphology after intra-articular injection of titanium dioxide nanoparticles in rats," Journal of Nanomaterials, vol. 2012, Article ID 452767, 11 pages, 2012.

[4] K. Riehemann, S. W. Schneider, T. A. Luger, B. Godin, M. Ferrari, and H. Fuchs, "Nanomedicine-challenge and perspectives," Angewandte Chemie, vol. 48, no. 5, pp. 872-897, 2009.

[5] C. Mason and P. Dunnill, "A brief definition of regenerative medicine," Regenerative Medicine, vol. 3, no. 1, pp. 1-5, 2008.

[6] O. Sadan, N. Shemesh, R. Barzilay et al., "stem cells induced to secrete neurotrophic factors attenuate quinolinic acid toxicity: a potential therapy for Huntington's disease," Experimental Neurology, vol. 234, no. 2, pp. 417-427, 2012.

[7] K. M. Krishnan, "Biomedical nanomagnetics: a spin through possibilities in imaging, diagnostics, and therapy," IEEE Transactions on Magnetics, vol. 46, no. 7, pp. 2523-2558, 2010.

[8] Q. A. Pankhurst, J. Connolly, S. K. Jones, and J. Dobson, “Applications of magnetic nanoparticles in biomedicine," Journal of Physics D, vol. 36, no. 13, pp. R167-R181, 2003.

[9] R. Banerjee, Y. Katsenovich, L. Lagos, M. Mciintosh, X. Zhang, and C. Z. Li, "Nanomedicine: magnetic nanoparticles and their biomedical applications," Current Medicinal Chemistry, vol. 17, no. 27, pp. 3120-3141, 2010.

[10] M. Mahmoudi, S. Sant, B. Wang, S. Laurent, and T. Sen, "Superparamagnetic iron oxide nanoparticles (SPIONs): development, surface modification and applications in chemotherapy," Advanced Drug Delivery Reviews, vol. 63, no. 1-2, pp. 24-46, 2011.

[11] A. Solanki, J. D. Kim, and K.-B. Lee, "Nanotechnology for regenerative medicine: nanomaterials for stem cell imaging," Nanomedicine, vol. 3, no. 4, pp. 567-578, 2008.

[12] Y. Jin, C. Jia, S.-W. Huang, M. O’Donnell, and X. Gao, "Multifunctional nanoparticles as coupled contrast agents," Nature Communications, vol. 1, no. 41, 2010.

[13] N. S. Barakat, "Magnetically modulated nanosystems: a unique drug-delivery platform," Nanomedicine, vol. 4, no. 7, pp. 799-812, 2009.

[14] M. M. Lin, D. K. Kim, A. J. El Haj, and J. Dobson, "Development of superparamagnetic iron oxide nanoparticles (SPIONS) for translation to clinical applications," IEEE Transactions on Nanobioscience, vol. 7, no. 4, pp. 298-305, 2008.

[15] A. Ito and M. Kamihira, "Tissue engineering using magnetite nanoparticles," Progress in Molecular Biology and Translational Science, vol. 104, pp. 355-395, 2011.

[16] L. Ferreira, "Nanoparticles as tools to study and control stem cells," Journal of Cellular Biochemistry, vol. 108, no. 4, pp. 746752, 2009.

[17] M. Mahmoudi, A. Simchi, M. Imani et al., "A new approach for the in vitro identification of the cytotoxicity of superparamagnetic iron oxide nanoparticles," Colloids and Surfaces B, vol. 75, no. 1, pp. 300-309, 2010.

[18] S. Hughes, A. J. El Haj, and J. Dobson, "Magnetic microand nanoparticle mediated activation of mechanosensitive ion channels," Medical Engineering and Physics, vol. 27, no. 9, pp. 754-762, 2005.

[19] S. Ju, G. Teng, Y. Zhang, M. Ma, F. Chen, and Y. Ni, "In vitro labeling and MRI of mesenchymal stem cells from human umbilical cord blood," Magnetic Resonance Imaging, vol. 24, no. 5, pp. 611-617, 2006.

[20] S. M. Cromer Berman, P. Walczak, and J. W. Bulte, "Tracking stem cells using magnetic nanoparticles," Wiley Interdisciplinary Reviews, vol. 3, no. 4, pp. 343-355, 2011.

[21] K.-S. Park, J. Tae, B. Choi et al., "Characterization, in vitro cytotoxicity assessment, and in vivo visualization of multimodal, RITC-labeled, silica-coated magnetic nanoparticles for labeling human cord blood-derived mesenchymal stem cells," Nanomedicine, vol. 6, no. 2, pp. 263-276, 2010.

[22] J. M. Hill, A. J. Dick, V. K. Raman et al., "Serial cardiac magnetic resonance imaging of injected mesenchymal stem cells," Circulation, vol. 108, no. 8, pp. 1009-1014, 2003.

[23] M. Getzlaff, Fundamentals of Magnetism, Springer, New York, NY, USA, 2008.

[24] M. Getzelt, Fundamentals of Magnetism, Springer, 2008.

[25] J. V. Frangioni and R. J. Hajjar, "In vivo tracking of stem cells for clinical trials in cardiovascular disease," Circulation, vol. 110, no. 21, pp. 3378-3383, 2004.

[26] M. Mahmoudi, H. Amiri, M. A. Shokrgozar et al., "Raman active jagged-shaped gold-coated magnetic particles as a novel multimodal nanoprobe," Chemical Communications, vol. 47, no. 37, pp. 10404-10406, 2011.

[27] A. K. Gupta and M. Gupta, "Synthesis and surface engineering of iron oxide nanoparticles for biomedical applications," Biomaterials, vol. 26, no. 18, pp. 3995-4021, 2005.

[28] H. Pardoe, W. Chua-anusorn, T. G. S. Pierre, and J. Dobson, "Structural and magnetic properties of nanoscale iron oxide particles synthesized in the presence of dextran or polyvinyl alcohol," Journal of Magnetism and Magnetic Materials, vol. 225, no. 1-2, pp. 41-46, 2001.

[29] J. W. M. Bulte, "In vivo MRI cell tracking: clinical studies," American Journal of Roentgenology, vol. 193, no. 2, pp. 314$325,2009$.

[30] C. C. Berry, "Possible exploitation of magnetic nanoparticlecell interaction for biomedical applications," Journal of Materials Chemistry, vol. 15, no. 5, pp. 543-547, 2005.

[31] H. S. Kim, S. Y. Oh, H. J. Joo, K.-R. Son, I.-C. Song, and W. K. Moon, "The effects of clinically used MRI contrast agents on the biological properties of human mesenchymal stem cells," NMR in Biomedicine, vol. 23, no. 5, pp. 514-522, 2010.

[32] Jasmin, A. L. M. Torres, H. M. P. Nunes et al., "Optimized labeling of bone marrow mesenchymal cells with superparamagnetic iron oxide nanoparticles and in vivo visualization by magnetic resonance imaging," Journal of Nanobiotechnology, vol. 9, article 4, 2011.

[33] M. Hofmann-Amtenbrink, H. Hofmann, and X. Montet, "Superparamagnetic nanoparticles-a tool for early diagnostics," Swiss Medical Weekly, vol. 140, pp. 7-13, 2010.

[34] M. Mahmoudi, H. Hosseinkhani, M. Hosseinkhani et al., "Magnetic resonance imaging tracking of stem cells in vivo using iron oxide nanoparticles as a tool for the advancement of clinical regenerative medicine," Chemical Reviews, vol. 111, no. 2, pp. 253-280, 2011.

[35] G. M. van Buul, E. Farrell, N. Kops et al., "Ferumoxidesprotamine sulfate is more effective than ferucarbotran for cell labeling: implications for clinically applicable cell tracking using MRI," Contrast Media and Molecular Imaging, vol. 4, no. 5, pp. 230-236, 2009.

[36] R. Zhou, P. D. Acton, and V. A. Ferrari, "Imaging stem cells implanted in infarcted myocardium," Journal of the American College of Cardiology, vol. 48, no. 10, pp. 2094-2106, 2006. 
[37] M. A. Mahmoudi and M. A. Shokrgozar, "Multifunctional stable fluorescent magnetic nanoparticles," Chemical Communications, vol. 48, no. 33, pp. 3957-3959, 2012.

[38] D. M. Huang, T. H. Chung, Y. Hung et al., "Internalization of mesoporous silica nanoparticles induces transient but not sufficient osteogenic signals in human mesenchymal stem cells," Toxicology and Applied Pharmacology, vol. 231, no. 2, pp. 208-215, 2008.

[39] T. H. Kim, J. K. Kim, W. Shim, S. Y. Kim, T. J. Park, and J. Y. Jung, "Tracking of transplanted mesenchymal stem cells labeled with fluorescent magnetic nanoparticle in liver cirrhosis rat model with 3-T MRI," Magnetic Resonance Imaging, vol. 28, no. 7, pp. 1004-1013, 2010.

[40] T. Kim, E. Momin, J. Choi et al., "Mesoporous silica-coated hollow manganese oxide nanoparticles as positive $\mathrm{T} 1$ contrast agents for labeling and MRI tracking of adipose-derived mesenchymal stem cells," Journal of the American Chemical Society, vol. 133, no. 9, pp. 2955-2961, 2011.

[41] K. J. Saldanha, R. P. Doan, K. M. Ainslie, T. A. Desai, and S. Majumdar, "Micrometer-sized iron oxide particle labeling of mesenchymal stem cells for magnetic resonance imagingbased monitoring of cartilage tissue engineering," Magnetic Resonance Imaging, vol. 29, no. 1, pp. 40-49, 2011.

[42] R. T. Castaneda, S. Boddington, T. D. Henning et al., "Labeling human embryonic stem-cell-derived cardiomyocytes for tracking with MR imaging," Pediatric Radiology, vol. 41, no. 11, pp. 1384-1392, 2011.

[43] G. J. R. Delcroix, M. Jacquart, L. Lemaire et al., "Mesenchymal and neural stem cells labeled with HEDP-coated SPIO nanoparticles: in vitro characterization and migration potential in rat brain," Brain Research, vol. 1255, pp. 18-31, 2009.

[44] A. Omidkhoda, H. Mozdarani, A. Movasaghpoor, and A. A. P. Fatholah, "Study of apoptosis in labeled mesenchymal stem cells with superparamagnetic iron oxide using neutral comet assay," Toxicology in Vitro, vol. 21, no. 6, pp. 1191-1196, 2007.

[45] P. G. Kyrtatos, P. Lehtolainen, M. Junemann-Ramirez et al., "Magnetic tagging increases delivery of circulating progenitors in vascular injury," Cardiovascular Interventions, vol. 2, no. 8, pp. 794-802, 2009.

[46] V. H. B. Ho, A. Barcza, R. Chen, K. H. Müller, N. J. Darton, and N. K. H. Slater, "The precise control of cell labelling with streptavidin paramagnetic particles," Biomaterials, vol. 30, no. 33, pp. 6548-6555, 2009.

[47] J. A. Kim, H.-J. Lee, H. J. Kang, and T. H. Park, "The targeting of endothelial progenitor cells to a specific location within a microfluidic channel using magnetic nanoparticles," Biomedical Microdevices, vol. 11, no. 1, pp. 287-296, 2009.

[48] J.-K. Hsiao, M.-F. Tai, H.-H. Chu et al., "Magnetic nanoparticle labeling of mesenchymal stem cells without transfection agent: cellular behavior and capability of detection with clinical 1.5 T magnetic resonance at the single cell level," Magnetic Resonance in Medicine, vol. 58, no. 4, pp. 717-724, 2007.

[49] M. Hoehn, E. Küstermann, J. Blunk et al., "Monitoring of implanted stem cell migration in vivo: a highly resolved in vivo magnetic resonance imaging investigation of experimental stroke in rat," Proceedings of the National Academy of Sciences of the United States of America, vol. 99, no. 25, pp. 16267-16272, 2002.

[50] S. M. C. Berman, P. Walczak, and J. W. M. Bulte, "Tracking stem cells using magnetic nanoparticles," Wiley Interdiscip Rev-Nanomed Nanobiotechnol, vol. 3, no. 4, pp. 343-355, 2011.
[51] S. Ramaswamy, J. B. Greco, M. C. Uluer et al., "Magnetic resonance imaging of chondrocytes labeled with superparamagnetic iron oxide nanoparticles in tissue-engineered cartilage," Tissue Engineering A, vol. 15, no. 12, pp. 3899-3910, 2009.

[52] I. Wimpenny, H. Markides, and A. El Haj, "Orthopaedic applications of nanoparticle-based stem cell therapies," Stem Cell Research \& Therapy, vol. 3, no. 2, p. 13, 2012.

[53] N. J. Sniadecki, "Minireview: a tiny touch: activation of cell signaling pathways with magnetic nanoparticles," Endocrinology, vol. 151, no. 2, pp. 451-457, 2010.

[54] S. H. Cartmell, J. Dobson, S. B. Verschueren, and A. J. El Haj, "Development of magnetic particle techniques for long-term culture of bone cells with intermittent mechanical activation," IEEE Transactions on Nanobioscience, vol. 1, no. 2, pp. 92-97, 2002.

[55] D. E. Ingber, "Cellular mechanotransduction: putting all the pieces together again," FASEB Journal, vol. 20, no. 7, pp. 811827, 2006.

[56] G. R. Kirkham, K. J. Elliot, A. Keramane et al., "Hyperpolarization of human mesenchymal stem cells in response to magnetic force," IEEE Transactions on Nanobioscience, vol. 9, no. 1, pp. 71-74, 2010.

[57] J. M. Kanczler, H. S. Sura, J. Magnay et al., "Controlled differentiation of human bone marrow stromal cells using magnetic nanoparticle technology," Tissue Engineering A, vol. 16, no. 10, pp. 3241-3250, 2010.

[58] S. H. Cartmell, J. Dobson, S. B. Verschueren, and A. J. El Haj, "Development of magnetic particle techniques for long-term culture of bone cells with intermittent mechanical activation," IEEE Transactions on Nanobioscience, vol. 1, no. 2, pp. 92-97, 2002.

[59] S.-W Cho, F. Yang, S. M. Son et al., "Therapeutic angiogenesis using genetically engineered human endothelial cells," Journal of Controlled Release, vol. 160, no. 3, pp. 515-524, 2012.

[60] M. C. Duran, S. Willenbrock, A. Barchanski et al., "Comparison of nanoparticle-mediated transfection methods for DNA expression plasmids: efficiency and cytotoxicity," Journal of Nanobiotechnology, vol. 9, article 47, 2011.

[61] A. Fouriki, M. A. Clements, N. Farrow, and J. Dobson, "Efficient transfection of MG-63osteoblasts using magnetic nanoparticles and oscillating magnetic fields," Journal of Tissue Engineering and Regenerative Medicine. In press.

[62] C.-W. Lu, Y. Hung, J.-K. Hsiao et al., "Bifunctional magnetic silica nanoparticles for highly efficient human stem cell labeling," Nano Letters, vol. 7, no. 1, pp. 149-154, 2007.

[63] C. K. Sung, K. A. Hong, S. Lin et al., "Dual-modal nanoprobes for imaging of mesenchymal stem cell transplant by MRI and fluorescence imaging," Korean Journal of Radiology, vol. 10, no. 6, pp. 613-622, 2009.

[64] "The dose makes the poison," Nature Nanotechnology, vol. 6, no. 6, p. 329, 2011.

[65] C.-Y. Yang, J.-K. Hsiao, M.-F. Tai et al., "Direct labeling of hMSC with SPIO: the long-term influence on toxicity, chondrogenic differentiation capacity, and intracellular distribution," Molecular Imaging and Biology, vol. 13, no. 3, pp. 443451, 2011.

[66] V. Dousset, T. Tourdias, B. Brochet, C. Boiziau, and K. G. Petry, "How to trace stem cells for MRI evaluation?" Journal of the Neurological Sciences, vol. 265, no. 1-2, pp. 122-126, 2008.

[67] M. K. Khaing Oo, Y. Yang, Y. Hu, M. Gomez, H. Du, and H. Wang, "Gold nanoparticle-enhanced and size-dependent generation of reactive oxygen species from protoporphyrin IX," ACS Nano, vol. 6, no. 3, pp. 1939-1947, 2012. 
[68] R. Weissleder, D. D. Stark, B. L. Engelstad et al., "Superparamagnetic iron oxide: pharmacokinetics and toxicity," American Journal of Roentgenology, vol. 152, no. 1, pp. 167-173, 1989.

[69] E. K. Schlachter, H. R. Widmer, A. Bregy et al., "Metabolic pathway and distribution of superparamagnetic iron oxide nanoparticles: in vivo study," International Journal of Nanomedicine, vol. 6, pp. 1793-1800, 2011.

[70] M. F. Kircher, S. S. Gambhir, and J. Grimm, "Noninvasive celltracking methods," Nature Reviews Clinical Oncology, vol. 8, no. 11, pp. 677-688, 2011.

[71] W. Strober, Trypan Blue Exclusion Test of Cell Viability. Current Protocols in Immunology, John Wiley \& Sons, 2001.

[72] B. C. Saravanan, C. Sreekumar, G. C. Bansal, D. Ray, J. R. Rao, and A. K. Mishra, "A rapid MTT colorimetric assay to assess the proliferative index of two Indian strains of Theileria annulata," Veterinary Parasitology, vol. 113, no. 3-4, pp. 211216, 2003.

[73] “TACS MTT Cell Proliferation Assays,” 2009, http://www.trevigen.com/docs/protocol_4890-XX-K.pdf.

[74] "LDH-CytotoxicityAssay Kit II (500 assays)," 2012, http:// www.abcam.com/LDH-Cytotoxicity-Assay-Kit-II-500-assaysab65393.pdf.

[75] "LIVE/DEAD Viability/Cytotoxicity Kit *for mammalian cells*," 2005, http://tools.invitrogen.com/content/sfs/manuals/mp03224.pdf.

[76] "BrdU Cell Proliferation Assay Kit," 2011, http://www.cellsignal.com/pdf/6813.pdf.

[77] S. Laurent, S. Dutz, U. O. Häfeli, and M. Mahmoudi, "Magnetic fluid hyperthermia: focus on superparamagnetic iron oxide nanoparticles," Advances in Colloid and Interface Science, vol. 166, no. 1-2, pp. 8-23, 2011.

[78] M. Mahmoudi, M. A. Sahraian, M. A. Shokrgozar, and S. Laurent, "Superparamagnetic iron oxide nanoparticles: promises for diagnosis and treatment of multiple sclerosis," ACS Chemical Neuroscience, vol. 2, no. 3, pp. 118-140, 2011.

[79] S. Sharifi, S. Behzadi, S. Laurent, M. Laird Forrest, P. Stroeve, and M. Mahmoudi, "Toxicity of nanomaterials," Chemical Society Reviews, vol. 41, no. 6, pp. 2323-2343, 2012.

[80] M. Lundqvist, J. Stigler, and T. Cedervall, "The evolution of the protein corona around nanoparticles: a test study," ACS Nano, vol. 5, no. 9, pp. 7503-7509, 2011.

[81] M. Mahmoudi, I. Lynch, M. R. Ejtehadi, M. P. Monopoli, F. B. Bombelli, and S. Laurent, "Protein-canoparticle interactions: opportunities and challenges," Chemical Reviews, vol. 111, no. 9, pp. 5610-5637, 2011.

[82] S. Laurent, C. Burtea, C. Thirifays, H. UO, and M. Mahmoudi, "Crucial ignored parameters on nanotoxicology: the importance of toxicity assay modifications and "cell vision"', Plos One, vol. 7, no. 1, Article ID e29997, 2012.

[83] M. Mahmoudi, S. Laurent, M. A. Shokrgozar, and M. Hosseinkhani, "Toxicity evaluations of superparamagnetic iron oxide nanoparticles: cell "vision" versus physicochemical properties of nanoparticles," ACS Nano, vol. 5, no. 9, pp. 72637276, 2011.

[84] X. Li, C. A. van Blitterswijk, Q. Feng, F. Cui, and F. Watari, "The effect of calcium phosphate microstructure on bonerelated cells in vitro," Biomaterials, vol. 29, no. 23, pp. 33063316, 2008.

[85] J. Huang, J. Wang, X. Su et al., "Biocompatibility of nanoporous $\mathrm{TiO}_{2}$ coating on NiTi alloy prepared via dealloying method," Journal of Nanomaterials, vol. 2012, Article ID 731592, 7 pages, 2012. 

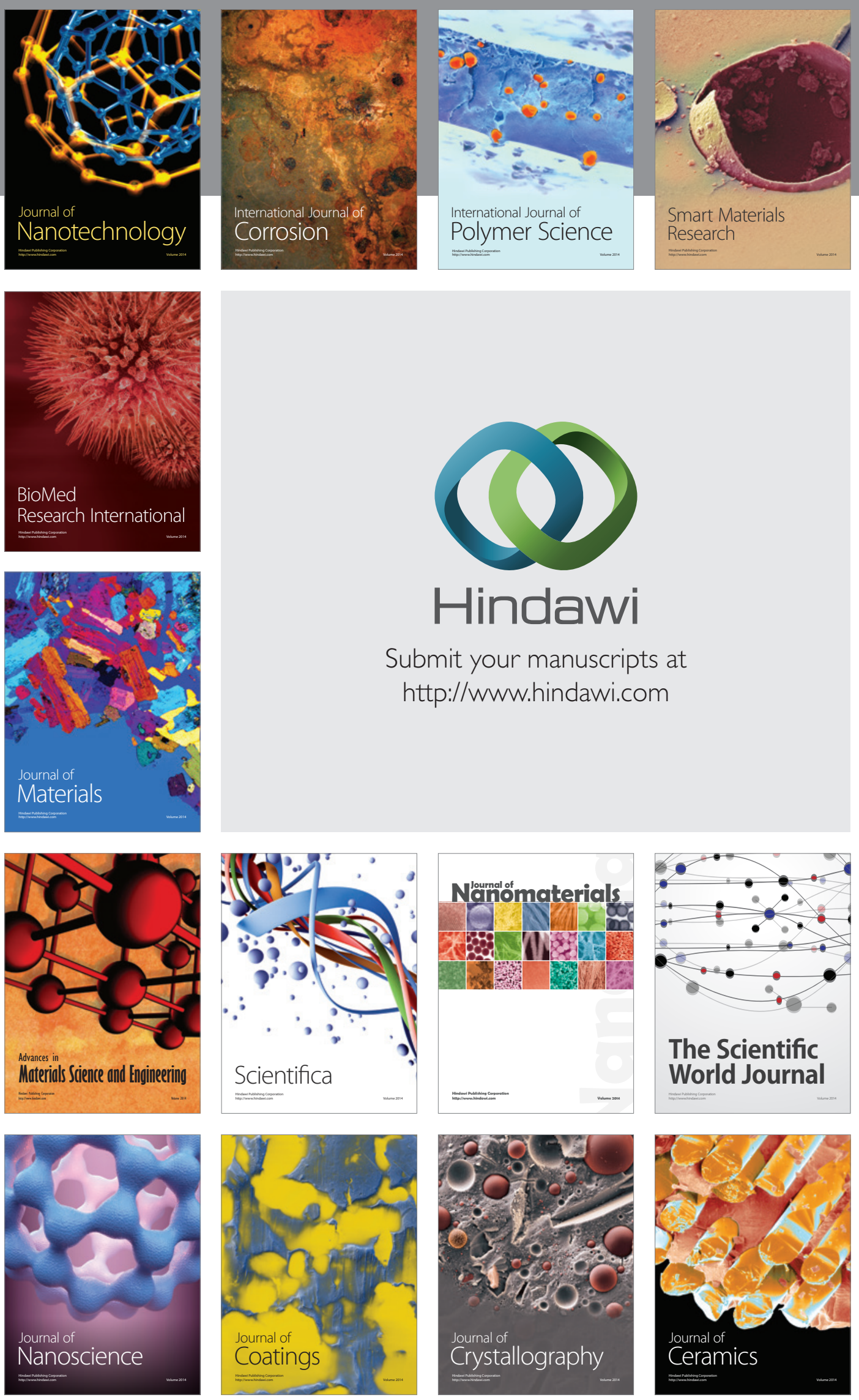

The Scientific World Journal

Submit your manuscripts at

http://www.hindawi.com

\section{World Journal}

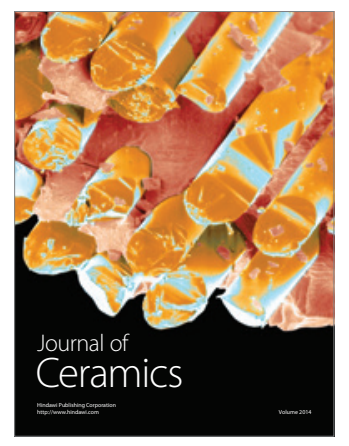

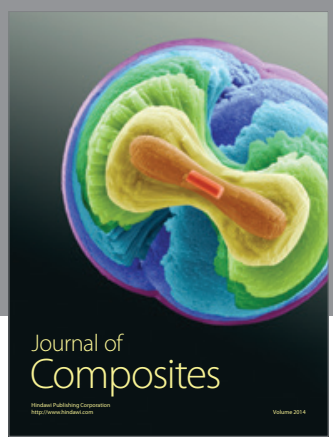
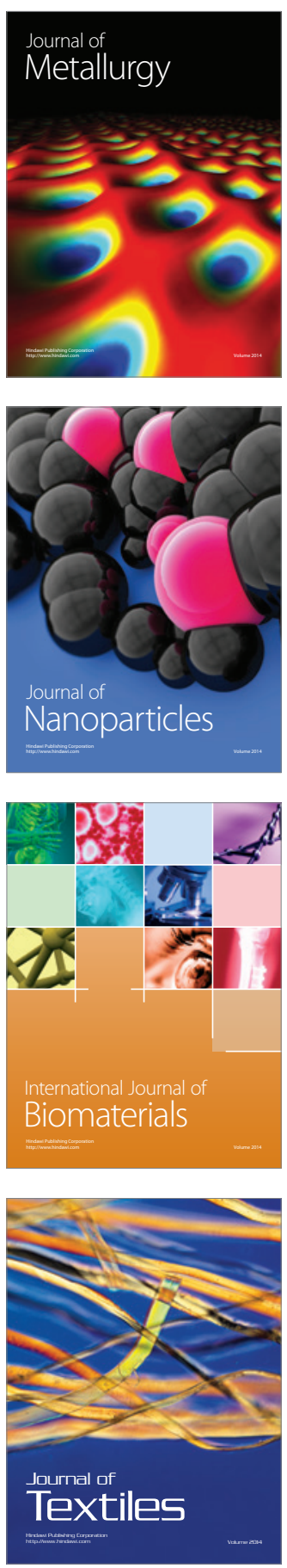